\title{
Soft Iterative Linear Detection for LDPC Coded MIMO Scheme with PSK
}

\author{
Meixiang Zhang1, Sooyoung Kim² \\ ${ }^{1}$ Yangzhou University, Yangzhou, China \\ ${ }^{2}$ Chonbuk National University, Jeonju, Korea \\ Email:maehyang@foxmail.com,sookim@jbnu.ac.kr
}

How to cite this paper: Zhang, M.X. and Kim, S. (2017) Soft Iterative Linear Detection for LDPC Coded MIMO Scheme with PSK. Int. J. Communications, Network and System Sciences, 10, 148-156. https://doi.org/10.4236/ijcns.2017.108B016

Received: May 27, 2017

Accepted: August 11, 2017

Published: August 14, 2017

\begin{abstract}
A number of study results demonstrated that the performance of the coded MIMO scheme can be highly enhanced by incorporating iterative decoding and detection scheme by exchanging soft information between the symbol detector and decoder. One of the critical problems of these iterative schemes is an exponential order of the complexity with increase of number of bits in a symbol and the number of antennas. In this paper, we present an efficient iterative detection and decoding scheme for MIMO systems with phase shift keying (PSK) modulation schemes and low density parity check (LDPC) codes. In order to reduce the complexity by the number of antennas, we adopt minimum mean square error (MMSE) based linear detection scheme with parallel interference cancellation. In addition, soft bit estimation is made only with a single distance calculation per bit, with approximating performance to the maximum likelihood detection ${ }^{1}$.
\end{abstract}

\section{Keywords}

MMSE, MIMO, Iterative Detection, Soft Bit Estimation, LDPC, PSK

\section{Introduction}

There have been a number of research studies on the development of detection schemes for multi-input multi-output (MIMO) systems, in order to achieve a capacity approaching performance. The basic idea is to utilize a detector that maximizes the a posteriori probability (MAP) in order to achieve the best performance in combination with powerful forward error correction (FEC) coding scheme. In addition to the iterative soft decoding of the FEC scheme, re-utilization of the soft output fed-back into the symbol detection process made

${ }^{1}$ This research was supported by the National Natural Science Foundation of China (No. 61601403), Universities Natural Science Research Project of Jiangsu Province (No. 16KJB510043). 
it possible to produce a capacity approaching performance [1]. However, a direct implementation of these iterative processes usually requires an exponentiallyincreasing computational complexity according to the number of antennas and the number of bits per symbol.

Recently, a number of researches reported results on minimum mean square error (MMSE) based MIMO detection schemes with soft iterative processes, due to their reasonable performance and complexity trade-offs [2] [3]. The parallel interference cancellation with MMSE (PIC-MMSE) MIMO detection schemes were proposed in order to enhance the performance as well as the computational efficiency compared to the conventional MMSE-based scheme [4] [5] [6]. These PIC-MMSE based detection schemes reduced the complexity of symbol-level detection to a linear-order. In addition to the symbol level detection, we need another step to extract soft bit information from the soft symbol values, and a direct implementation of this step requires an exponential order of complexity with increase in the number of bits in a symbol.

In this paper, we present an efficient linear MIMO detection scheme for a coded MIMO system, where phase shift keying (PSK) modulation schemes are used with low density parity check (LDPC) codes. In the proposed scheme, soft symbol values are first estimated by utilizing a PIC-MMSE filter, and then soft bit information (SBI) values are estimated only with a single distance estimation per bit. For this, we first normalize the detected symbol from the PIC-MMSE filtering process, and then map it to a specific region, so that SBI estimation can be made with a single distance calculation [7]. By this way, overall complexity is in a linear order, and thus it can be easily applied to a massive MIMO system, i.e., even the number of antennas are greater than a few tens.

The remainder of this paper is organized as follows. Section II describes a MIMO system model with soft iterative detection and decoding (IDD), with an FEC scheme with soft iterative decoding process. Next, the operational principles of the PIC-MMSE MIMO detection for IDD are described. In Section III, we detail the SBI estimation process by describing mathematical formulas when the PIC-MMSE detector is employed with PSK modulation. Section IV demonstrates the bit error rate (BER) performance and the complexity of the proposed methods are compared with the conventional schemes for the LDPC coded MIMO systems with PSK modulation schemes. Finally, conclusions are drawn in Section V.

\section{PIC-MMSE Detection for a Coded MIMO System}

\subsection{System Model}

Figure 1 shows the block diagram for an $N_{t} \times N_{r}$ MIMO system with soft IDD, where a LDPC code is used as an FEC scheme and a PSK is used for modulation. At the transmitter, the bit information vector $u$ is encoded to produce codeword $c$. Then $N_{t} \cdot K$ codewords are interleaved and modulated successively before they are mapped to the $N_{t}$ transmitted antennas, where $K$ is the number of bits per transmitted symbol. The interleaved bits 


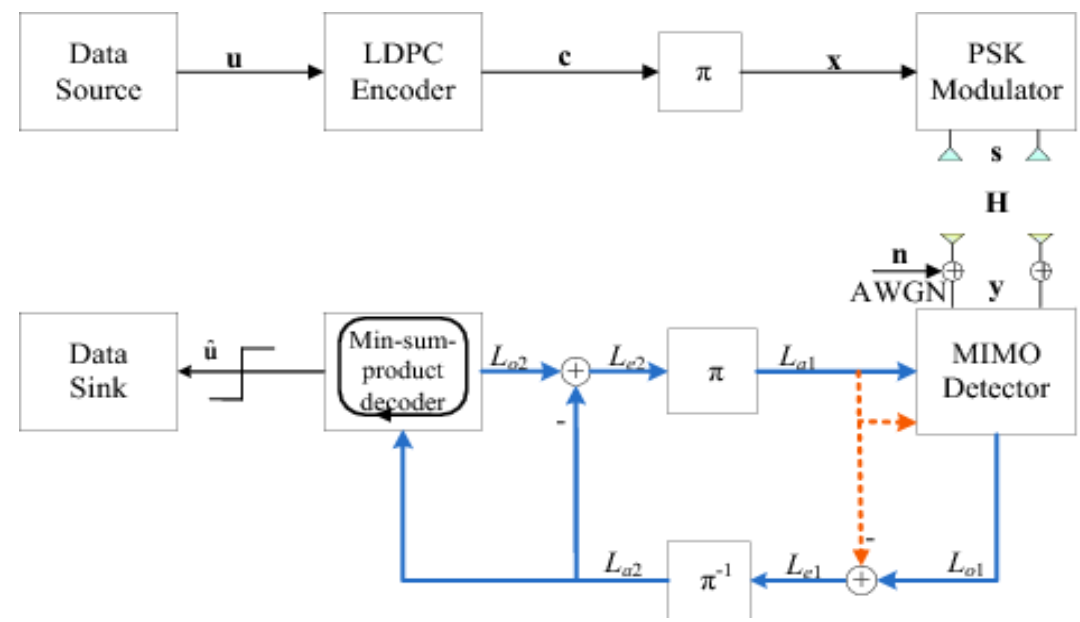

Figure 1. Coded MIMO system model with soft IDD.

$x=\left[x_{1,1}, \cdots, x_{1, K}, x_{2,1}, \cdots, x_{m, k}, \cdots, x_{N_{t}, K}\right]$ are divided and modulated to transmitted symbol vector $s=\left[s_{1}, s_{2}, \cdots, s_{m}, \cdots, s_{N_{t}}\right]^{T}$, where $x_{m, k}$ represents the $k$ th bit of the $m$ th symbol $s_{m}$ which is independently chosen from a complex constellation $O$.

Suppose the received symbol vector is represented as $\mathbf{y}=\left[y_{1}, y_{2}, \cdots, y_{N_{r}}\right]^{T}$, and a flat Rayleigh fading channel model is chosen as the MIMO channel, i.e., $\mathbf{H}=\left[h_{i, j}\right]_{N_{r} \times N_{t}}$, where $h_{i, j}$ denotes the channel fading coefficient between the th transmitted antenna and the th received antenna. The elements of the channel matrix are modeled by independent and complex-valued Gaussian random variables with zero mean and unit variance. Then,

$$
\mathbf{y}=\mathbf{H s}+\mathbf{n},
$$

where $\mathbf{n}$ is an $N_{r} \times 1$ vector whose elements are independent zero-mean complex Gaussian random variables with variance $\sigma^{2}$ per dimension.

Figure 1 shows the soft IDD procedure. The SBI of the MIMO detector, $L_{o 1}$ is first calculated and subtracted by the a priori information of the detector $L_{a 1}$, i.e., $L_{e 1}=L_{o 1}-L_{a 1}$; then the extrinsic information $L_{e 1}$ is passed through the de-interleaver, and its de-interleaved version $L_{a 2}$ is utilized by the channel decoder as the a priori information. The channel decoder estimates the information sequence and generates its soft output $L_{o 2}$, by using soft iterative decoding algorithm such as the min-sum-product algorithm. Subsequently, the interleaved version of extrinsic information, $L_{e 2}=L_{o 2}-L_{a 2}$ is fed back to the detector as the a priori information.

\subsection{Soft Iterative PIC-MMSE Detection}

The PIC-MMSE MIMO detector is performed as follows. First, with the a priori information, $L_{a 1}$ from the channel decoder (at the first iteration it is set to zero), the expected value of the th transmitted symbol, $\widetilde{s}_{i}$ is calculated as follows [8]:

$$
\widetilde{s_{i}}=\sum_{a \in O} \frac{a}{2^{K}} \prod_{k=1}^{K}\left(1+\tilde{x}_{i, k} \tanh \frac{L_{a 1}\left(x_{i, k}\right)}{2}\right),
$$


where $a$ is a constellation symbol from $O$, and $\tilde{x}_{i, k}$ is set to be -1 and 1 according to the $k$ th bit of $a$ is 0 and 1 , respectively. At the same time, the variance of the $i$ th transmitted symbol, $E_{i}$ is calculated by:

$$
E_{i}=\sum_{a \in O} \frac{|a|^{2}}{2^{K}} \prod_{k=1}^{K}\left(1+\tilde{x}_{i, k} \tanh \frac{L_{a 1}\left(x_{i, k}\right)}{2}\right)-\left|\tilde{s}_{i}\right|^{2} .
$$

The second step is PIC on the received symbol vector, $\mathbf{y}$, along with MMSE filtering. The PIC process on $\mathbf{y}$ is performed using the estimated expectation, $\widetilde{s_{i}}$ in (2) as follows:

$$
\hat{\mathbf{y}}_{i}=\mathbf{y}-\sum_{j \neq i} \mathbf{h}_{j} \widetilde{s_{j}}=\mathbf{h}_{i} s_{i}+\tilde{\mathbf{n}},
$$

where $\hat{y}_{i}$ is the interference canceled symbol for the $i$ th layer, $\mathbf{h}_{i}$ is the $i$ th column vector of the channel matrix $\mathbf{H}$, and $\tilde{\mathbf{n}}$ denotes the residual noise plus interference (NPI) term expressed by $\tilde{\mathbf{n}}=\sum_{j \neq i} \mathbf{h}_{j}\left(s_{i}-\tilde{s}_{i}\right)+\mathbf{n}$. At the same time, the MMSE filtering process is performed as follows:

$$
\mathbf{W}^{H}=\mathbf{H}^{H}\left(\mathbf{H} \Lambda \mathbf{H}^{H}+\sigma^{2} \mathbf{I}_{N_{r}}\right)^{-1},
$$

where $\Lambda$ is a diagonal matrix with $\lambda_{i, i}=E_{i}$ estimated in (3).

The third step is suppressing the NPI term in (4) using the MMSE filter in (5). Then, the filtered result for the $i$ th layer, i.e., the estimated value of the $i$ th symbol, can be expressed as:

$$
\hat{z}_{i}=\mathbf{w}_{i}^{H} \mathbf{y}_{i},
$$

where $\mathbf{w}_{i}^{H}$ denotes the $i$ th row of $W^{H}$ and corresponds to the MMSE filter for the $i$ th layer. The last step is to calculate SBI contained in $\hat{z}_{i}$. Let us denote SBI of the kth bit contained in the ith symbol, in terms of log-likelihood ratio (LLR) estimated on the observation of $\mathbf{y}$ over the channel $\mathbf{H}$, as $L_{o 1}\left(x_{i, k} \mid \mathbf{y}, \mathbf{H}\right)$, then

$$
\begin{aligned}
& L_{o 1}\left(x_{i, k} \mid \mathbf{y}, \mathbf{H}\right) \approx L_{o 1}\left(x_{i, k} \mid \hat{z}_{i}\right) \\
& \approx \min _{a \in O_{k}^{0}}\left\{\rho_{i}\left|z_{i}-a\right|^{2}+F_{i, k}\right\}-\min _{a \in O_{k}^{1}}\left\{\rho_{i}\left|z_{i}-a\right|^{2}+F_{i, k}\right\},
\end{aligned}
$$

where $a \in O_{k}^{0}$ and $a \in O_{k}^{1}$ denote constellation symbols with the $k$ th bit of 0 and 1 , respectively, and

$$
\begin{aligned}
& F_{i, k}=\sum_{k=1}^{K} \ln \left(1+e^{\left(-x_{i, k} L_{a 1}\left(x_{i, k}\right)\right)}\right), \\
& Z_{i}=\hat{z}_{i} / \mathbf{w}_{i}^{H} \mathbf{h}_{i}, \\
& \rho_{i}=\mathbf{w}_{i}^{H} \mathbf{h}_{i} /\left(1-E_{i} \mathbf{w}_{i}^{H} \mathbf{h}_{i}\right) .
\end{aligned}
$$

It is clear in (7) that the search process to find the solution of $\min (\cdot)$ needs complexity of $O\left(2^{K}\right)$ for every layer.

\section{Proposed Scheme}

\subsection{SBI Estimation Using Symbol Mapping of PSK}

It was reported that there were no performance degradation even if the a priori information from the channel decoder in (7) was neglected, for the systems 
using binary phase shift keying (BPSK) and quadrature phase shift keying (QPSK) [9]. In this paper, we use this fact, and assume that the a priori information, $L_{a 1}$ can be neglected, then (7) can be simplified as follows.

$$
L_{o 1}\left(x_{i, k} \mid \mathbf{y}, \mathbf{H}\right) \approx \rho_{i}\left(\min _{a \in O_{k}^{0}}\left(\left|z_{i}-a\right|^{2}\right)-\min _{a \in O_{k}^{1}}\left(\left|z_{i}-a\right|^{2}\right)\right) .
$$

With this simplification, the argument of $\min (\cdot)$ operations in (9) is now $z_{i}$. In other words, we need to find a symbol, $a$ with the minimum distance from $z_{i}$ for the $k$ th bit. For this purpose, we map $z_{i}$ to a target unit range where there is only one constellation symbol to estimate the distance from $z_{i}$. We utilize the symbol mapping technique using the symmetric characteristics of PSK symbol constellation for a given bit [7].

First, we consider that the constellation diagram can be divided into $2^{K}$ unit regions for $2^{K}$-ary PSK modulation scheme, using the bisectors between the constellation symbols. Subsequently, $z_{i}$ is recursively mapped to a specific region in the first quadrant to $\left|z_{i}\right| e^{j \varepsilon_{k}^{b}}$, where $\varepsilon_{k}^{b}, b \in\{0,1\}$ is the phase of the mapped symbol used for the $k$ th bit of $b$. Then, at the ith layer, $\left|z_{i}-a\right|^{2}$ for the corresponding bit of $a$ is the same as the distance between $\left|z_{i}\right| e^{j \varepsilon_{k}^{b}}$ and a constellation symbol located in the same unit region as $\left|z_{i}\right| e^{j \varepsilon_{k}^{b}}$. The mapped symbol, $\left|z_{i}\right| e^{j \varepsilon_{k}^{b}}$ and the corresponding constellation symbol, are determined by the symmetric characteristics of the symbol constellation, based on Euclidean geometry for a given bit.

With the above property, (9) can be generalized as

$$
\begin{aligned}
& L_{o 1}\left(x_{i, k} \mid \mathbf{y}, \mathbf{H}\right) \\
& \approx \mu_{i, k}\left\{\left|z_{i}\right| e^{j \varepsilon_{k}^{b}}-\left.q_{k}^{0}\right|^{2}-|| z_{i}\left|e^{j \varepsilon_{k}^{b}}-q_{k}^{1}\right|^{2}\right\},
\end{aligned}
$$

where $\mu_{i, k}$ is a scaling parameter for the $k$ th bit of the th layer, which is a function of $\rho_{i}$ by reflecting the sign change introduced by the symbol mapping, and $q_{k}^{b}$ are the unique symbols nearest to $\left|z_{i}\right| e^{j \varepsilon_{k}^{b}}$, i.e., the mapped version of the detected symbol, with a phase of $\varepsilon_{k}^{b}$.

In addition, $\varepsilon_{k}^{b}$ and $q_{k}^{b}$ are determined prior to the system implementation, and they are constant values independent of $z_{i}$. For PSK with the Graymapping, $q_{k}^{b}$ and $\varepsilon_{k}^{b}$ can be found as shown in Table 1 and Table 2. In 2, $\theta_{i}$ values can be recursively found as follows

$$
\theta_{k}=\pi / 2^{k+1}-\left|\pi / 2^{k+1}-\theta_{k}\right|,
$$

where $\theta_{0}=\tan ^{-1}\left(\left|\Im\left(z_{i}\right)\right| /\left|\Re\left(z_{i}\right)\right|\right)$. In this way, the search process to find the minima can be eliminated. Thus, the complexity to estimate SBI is reduced to

Table 1. $q_{k}^{b}$ values for $2^{K}$-ary PSK.

\begin{tabular}{ccc}
\hline$K$ & $q_{k}^{0}$ & $q_{k}^{1}$ \\
\hline$K=1$ & $e^{j \pi / K}$ & $e^{j(\pi / 2+\pi / K)}$ \\
$K=2$ & $e^{j \pi / K}$ & $e^{j(-\pi / K)}$ \\
$K>2$ & $e^{j \pi / K}$ & $e^{j\left(\pi / 2^{i-1}+\pi / K\right)}$ \\
\hline
\end{tabular}


Table 2. $\varepsilon_{k}^{b}$ values for $2^{K}$-ary PSK.

\begin{tabular}{ccc}
\hline$K$ & $\varepsilon_{k}^{0}$ & $\varepsilon_{k}^{1}$ \\
\hline$K=1$ & $\theta_{K-2}$ & $\theta_{0}$ \\
$K=2$ & $\theta_{K-2}$ & $\theta_{0}$ \\
$K>2$ & $\theta_{K-2}$ & $\theta_{k-2}$ \\
\hline
\end{tabular}

linear-order for each independent layer without degrading the performance from the Max-approach.

\subsection{Soft Symbol Estimation for PSK}

Soft symbol value estimations specified in (2) and (3) require $(K+1) 2^{K}$ times of multiplications, which exponentially increases by $K$. We reduce the complexities of computing (2) and (3), by finding the symbol values of $a$. We modify the idea reported in [9] for QAM.

By denoting $p_{i, k}$ as the probability of the $k$ th bit of the $i$ th layer to be 0 , the following equation applies:

$$
p_{i, k}=\operatorname{Pr}\left[x_{i, k}=0\right]=\frac{1}{2}\left(1-\zeta_{i, k}\right),
$$

where $\zeta_{i, k}=\tanh \frac{L_{a 1}\left(x_{i, k}\right)}{2}$, then,

$$
\frac{1}{2}\left(1+\zeta_{i, k}\right)=1-p_{i, k}
$$

With this information, we simplify the expressions of $\tilde{s}_{i}$ and $E_{i}$ for PSK schemes, so that they can be used in real-number operations; First, in the case of BPSK, $\tilde{s}_{i}$ and $E_{i}$ can be derived as follows:

$$
\begin{gathered}
\tilde{s}_{i}=\sum_{a \in O} \frac{a}{2^{K}} \prod_{k=1}^{K}\left(1+\tilde{x}_{i, k} \zeta_{i, k}\right) \\
=p_{i, 1}-\left(1-p_{i, 1}\right)=2 p_{i, 1}-1, \\
E_{i}=\sum_{a \in O} \frac{|a|^{2}}{2^{K}} \prod_{k=1}^{K}\left(1+\tilde{x}_{i, k} \zeta_{i, k}\right)-\left|\tilde{s}_{i}\right|^{2} \\
=\left[p_{i, 1}+\left(1-p_{i, 1}\right)\right]-\left|\tilde{s}_{i}\right|^{2}=1-\left|\tilde{s}_{i}\right|^{2},
\end{gathered}
$$

Then, we apply the same rule for the soft IDD scheme, and denote the iteration index inside the PIC-MMSE detector as $\alpha$, then (12) can be represented by:

$$
p_{i, k}^{\alpha}=\frac{1}{2}\left(1-\zeta_{i, k}^{\alpha}\right) .
$$

Equation (16) is then applied to QPSK, whereby QPSK can be decomposed into two independent BPSK. Then, expressions of the real and imaginary parts of the soft symbol values, $\mathfrak{R}\left(\tilde{S}_{i}^{\alpha}\right)$ and $\mathfrak{I}\left(\tilde{S}_{i}^{\alpha}\right)$ are formulated independently for QPSK with amplitude of $\Re_{1}=\cos (\pi / 4)$ and $\mathfrak{J}_{1}=\sin (\pi / 4)$, respectively, as follows: 


$$
\mathfrak{R}\left({\tilde{S_{i}}}_{i}^{\alpha}\right)=\mathfrak{R}_{1} \cdot\left(2 p_{i, 1}^{\alpha}-1\right), \mathfrak{I}\left(\tilde{s}_{i}^{\alpha}\right)=\mathfrak{J}_{1} \cdot\left(2 p_{i, 2}^{\alpha}-1\right),
$$

and accordingly, the $E_{i}^{\alpha}$ of QPSK can be simplified according to the following equation:

$$
E_{i}^{\alpha}=1-\left|\tilde{S}_{i}^{\alpha}\right|^{2}=1-\left(\Re^{2}\left(\tilde{S}_{i}^{\alpha}\right)+\mathfrak{I}^{2}\left(\tilde{S}_{i}^{\alpha}\right)\right) .
$$

The expressions for the real and imaginary part of $\tilde{\sim}_{i}^{\alpha}$ for 8-PSK can be derived as follows:

$$
\begin{aligned}
& \mathfrak{R}\left(\tilde{s}_{i}^{\alpha}\right)=\left(2 p_{i, 1}^{\alpha}-1\right)\left[\Re_{1} p_{i, 3}^{\alpha}+\mathfrak{I}_{1}\left(1-p_{i, 3}^{\alpha}\right)\right], \\
& \mathfrak{I}\left(\tilde{S}_{i}^{\alpha}\right)=\left(2 p_{i, 2}^{\alpha}-1\right)\left[\Re_{1}\left(1-p_{i, 3}^{\alpha}\right)+\mathfrak{I}_{1} p_{i, 3}^{\alpha}\right],
\end{aligned}
$$

where $\mathfrak{R}_{1}=\cos (\pi / 8)$ and $\mathfrak{I}_{1}=\sin (\pi / 8) . \mathfrak{R}\left(\tilde{S}_{i}^{\alpha}\right)$ and $\mathfrak{I}\left(\tilde{S}_{i}^{\alpha}\right)$ for the 16-PSK can be derived with the same principle, and formulized as follows

$$
\begin{aligned}
& \mathfrak{R}\left(\tilde{S}_{i}^{\alpha}\right) \\
& =\left(2 p_{i, 1}^{\alpha}-1\right)\left[\mathfrak{R}_{1} p_{i, 3}^{\alpha} p_{i, 4}^{\alpha}+\mathfrak{R}_{2} p_{i, 3}^{\alpha}\left(1-p_{i, 4}^{\alpha}\right)\right. \\
& \left.+\mathfrak{I}_{2}\left(1-p_{i, 3}^{\alpha}\right)\left(1-p_{i, 4}^{\alpha}\right)+\mathfrak{I}_{1}\left(1-p_{i, 3}^{\alpha}\right) p_{i, 4}^{\alpha}\right], \\
& \mathfrak{I}\left(\tilde{S}_{i}^{\alpha}\right) \\
& =\left(2 p_{i, 2}^{\alpha}-1\right)\left[\mathfrak{I}_{1} p_{i, 3}^{\alpha} p_{i, 4}^{\alpha}+\mathfrak{I}_{2} p_{i, 3}^{\alpha}\left(1-p_{i, 4}^{\alpha}\right)\right. \\
& \left.+\mathfrak{R}_{2}\left(1-p_{i, 3}^{\alpha}\right)\left(1-p_{i, 4}^{\alpha}\right)+\mathfrak{R}_{1}\left(1-p_{i, 3}^{\alpha}\right) p_{i, 4}^{\alpha}\right],
\end{aligned}
$$

where $\mathfrak{R}_{1}=\cos (\pi / 16), \mathfrak{J}_{1}=\sin (\pi / 16), \mathfrak{R}_{2}=\cos (3 \pi / 16)$ and $\mathfrak{I}_{2}=\sin (3 \pi / 16)$. With the same principle, the $\tilde{s}_{i}^{\alpha}$ in (2) for the higher-order PSK can be expanded.

\section{Simulation Results}

We simulated the BER performance of the proposed methods over a Rayleigh fading channel. We used the LDPC code with an information length of 16,200 bits and a code rate of $1 / 2$. The min-sum product algorithm with a correction factor was used [10]. The maximum iterations of the LDPC decoding was set to 10 , and the number of iterations between the LDPC decoder and PIC-MMSE detector was set to 4. As the conventional scheme, we employ (2) and (3) for soft symbol estimations inside PIC-MMSE and (7) for SBI estimation. On the other hand, in our proposed method, we utilized (17) to (22) for soft symbol estimations and (10) for SBI estimation.

Figure 2 and Figure 3 show the BER performance comparisons between the conventional and proposed schemes for $8 \times 8$ and $16 \times 16$ MIMO systems, respectively. As shown in the figures, the proposed method produces exactly the same performance with the conventional scheme, regardless of the modulation order and the number of antennas. On the other hand, the computational complexity of the proposed method is greatly reduced to almost linear-order, resulting from the elimination of search process to find the minima in the SBI calculation and a simplified soft symbol estimations. Table 3 demonstrates these. 


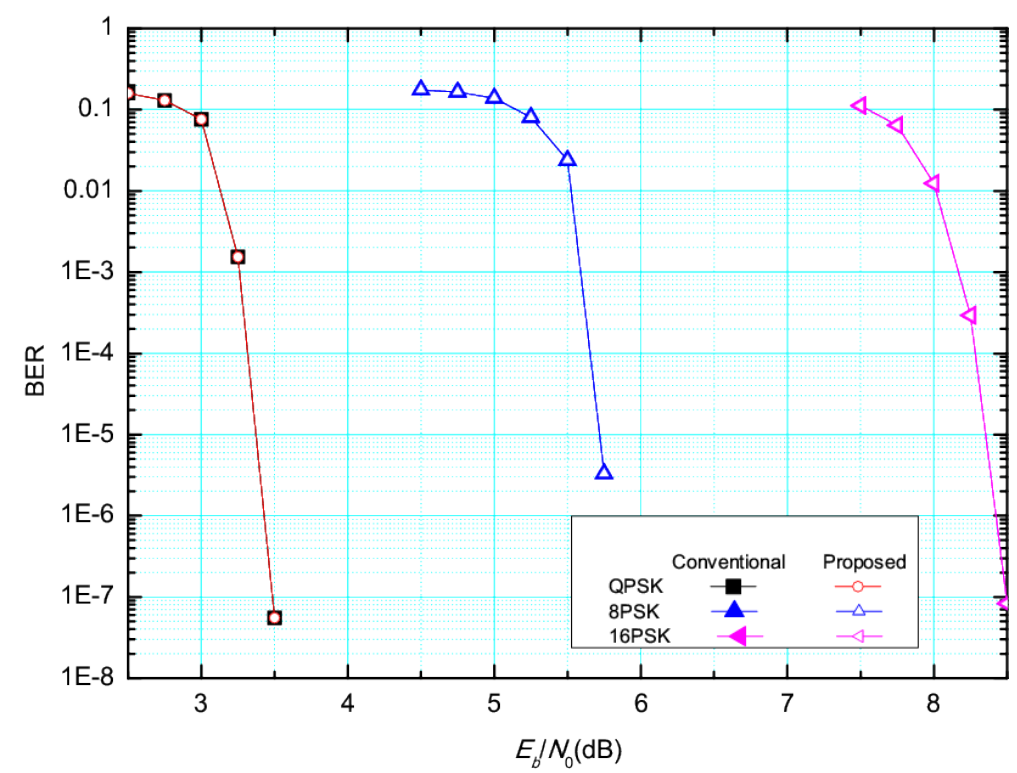

Figure 2. BER Performance comparison of $8 \times 8$ LDPC coded MIMO systems.

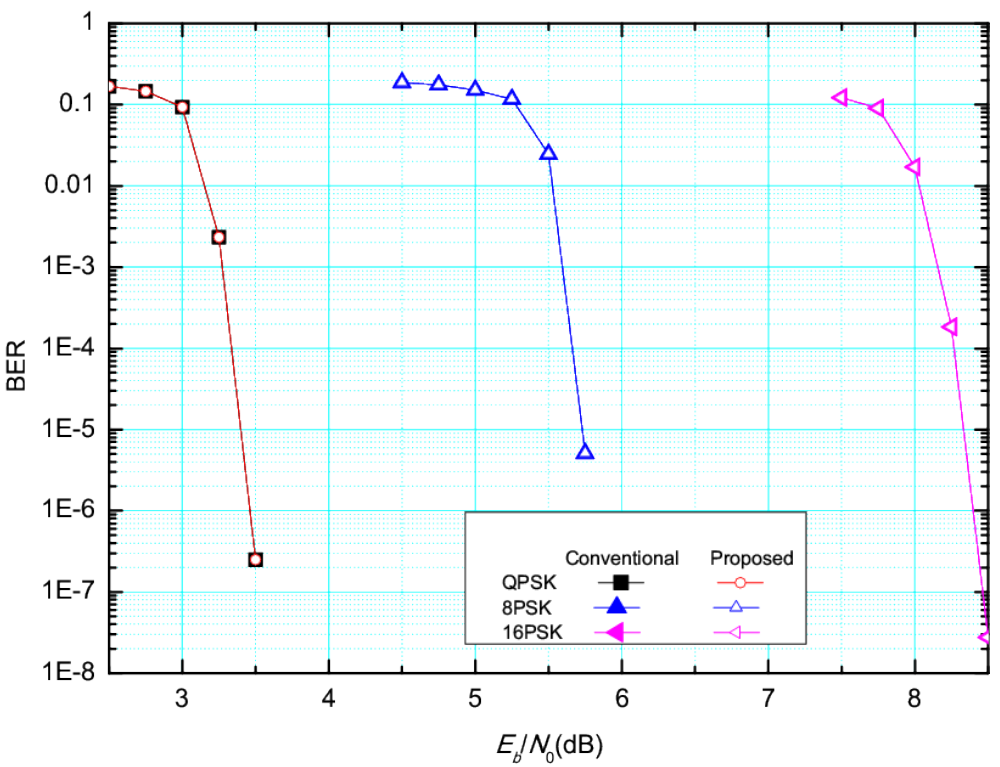

Figure 3. BER Performance comparison of $16 \times 16$ LDPC coded MIMO systems.

Table 3. Number of multiplications and additions to estimate $\tilde{S}_{i}$ and $E_{i}$ per PICMMSE detection.

\begin{tabular}{ccc}
\hline$K$ & $\tilde{s}_{i}$ & $E_{i}$ \\
\hline 1 & Proposed/conventional & Proposed/conventional \\
\hline 2 & $7 / 10$ & $4 / 20$ \\
3 & $14 / 40$ & $4 / 68$ \\
4 & $26 / 120$ & $4 / 196$ \\
\hline
\end{tabular}




\section{Conclusion}

In this paper, we proposed the symbol mapping technique for the PIC-MMSE based MIMO detection of PSK to reduce the complexity, resulting from the elimination of the search process to find the minima in the SBI estimation. To further reduce the computational complexity, we presented efficient method for PSK schemes to calculate the soft symbols in the PIC process. Simulation results showed that the proposed techniques reduced the complexity to nearly linearorder without degrading the BER performance.

\section{References}

[1] Hockwald, B.M. and Ten Brink, S. (2003) Achieving Near-Capacity on a Multiple-Antenna Channel. IEEE Transactions on Communication, 51, 389-399. https://doi.org/10.1109/TCOMM.2003.809789

[2] Ahmed, S. and Kim, S. (2015) Efficient Soft Bit Estimation for Joint Iterative Multiple-Input Multiple-Output Detection. IET Communications, 9, 2107-2113. https://doi.org/10.1049/iet-com.2015.0225

[3] Ahmed, S. and Kim, S. (2017) Efficient SIC-MMSE MIMO Detection with Three Iterative Loops. International Journal of Electronics and Communications, 72, 65 71. https://doi.org/10.1016/j.aeue.2016.11.015

[4] Tomasoni, A., Ferrari, M., Gatti, D., Osnato, F. and Bellini, S. (2006) Low Complexity Turbo MMSE Receiver for W-LAN MIMO Systems. Proc. of ICC 2006, 9, 4119 4124 .

[5] Bensaad, A., Bensaad, Z., Soudini, B. and Beloufa, A. (2013) SISO PIC-MMSE Detector in MIMO-OFDM Systems. Int. J. Mod. Eng. Research, 3, 2840-2847.

[6] Kuang, S.L., Ni, Z. and Lu, J. (2014) Low-Complexity Iterative Detection for Large-Scale Multiuse MIMO-OFDM Systems Using Approximate Message Passing. IEEE Journal on Selected Topics Signal Processing, 8, 902-915. https://doi.org/10.1109/JSTSP.2014.2313766

[7] Zhang, M. and Kim, S. (2016) Universal Soft Demodulation Schemes for M-Ary PSK and QAM. IET Communications, 10, 316-326. https://doi.org/10.1049/iet-com.2015.0730

[8] Zhang, M., Li, C., Kim, S. and Ahmed, S. (2016) Low Complexity Iterative PIC-MMSE Based Detection with Symbol Mapping. Proceedings of IEEE/CIC 2016, Chengdu, China, 27-29 July 2016. https://doi.org/10.1109/ICCChina.2016.7636778

[9] Fateh, S. (2009) VLSI Implementation of Soft-Input Soft-Output MMSE Parallel Interference Cancellation. Master's Thesis, ETH Zurich, Integrated Systems Laboratory.

[10] Eroz, M., Sun, F. and Lee, L. (2004) DVB-S2 Low Density Parity Check Codes with Near Shannon Limit Performance. International Journal of Satellite Communications and Networking, 22, 269-279. https://doi.org/10.1002/sat.787 
Submit or recommend next manuscript to SCIRP and we will provide best service for you:

Accepting pre-submission inquiries through Email, Facebook, LinkedIn, Twitter, etc. A wide selection of journals (inclusive of 9 subjects, more than 200 journals)

Providing 24-hour high-quality service

User-friendly online submission system

Fair and swift peer-review system

Efficient typesetting and proofreading procedure

Display of the result of downloads and visits, as well as the number of cited articles Maximum dissemination of your research work

Submit your manuscript at: http://papersubmission.scirp.org/

Or contact ijcns@scirp.org 